\title{
25. PROTOSTARS AND OTHER NEUTRAL CONDENSATIONS IN HII REGIONS
}

Introductory Report

(Thursday, September 18, 1969)

P. G. MEZGER

National Radio Astronomy Observatory*, Green Bank, W.Va., U.S.A.

and

Max-Planck-Institut für Radioastronomie, Bonn, Germany

\section{HII Regions and the Formation of Stars}

I have been invited to report on globules and $\mathrm{OH}$ emission sources. However, I will extend the topic to protostars and other condensations in $\mathrm{H}$ II regions, of which globules and $\mathrm{OH}$ emission sources are only special, although rather conspicuous, objects.

To comply with the wishes of the program committee, I will not enter into a general discussion of HII regions. However, in Section 2 I will mention some high-angularresolution radio observations that bear upon the original density distribution of the collapsing protostar or protocluster. Although I make this restriction, I want to stress that it would be very unfortunate if the theoreticians were left with the impression that there is good agreement between theory and observations of HII regions. Let me just point out one example which came up in several discussions at this meeting, i.e., the acceleration of neutral interstellar matter by expanding $\mathrm{H}$ II regions which surround $\mathrm{O}$ stars. The theory of this process has been meticulously worked out. However, there is little observational evidence to date of uniformly expanding H II regions and no evidence of thin, dense shells of neutral matter surrounding them, and to me the reality of this particular mechanism seems highly doubtful.

With this in mind, let me go on to the problem of star formation where the discrepancy between theory and observations appears to be even larger. The objects which will be discussed in this paper are, in one way or another, related to the process of star formation. How this process works in detail is still quite unclear, but the end result we know: stars with masses ranging from $0.08 M_{\odot}$ to about $50 M_{\odot}$. More massive stars apparently become unstable; less massive stars never attain a high enough central temperature for nuclear reactions to set in. Averaged over a larger volume of space, the mass spectrum of stars attains a maximum at about $0.5 M_{\odot}$. It decreases slowly in the direction of low mass stars but cuts off rapidly towards more massive stars. On the average only about 3 per cent of the total mass of all newly formed stars is contained in $\mathrm{O}$ stars. Nevertheless, these $\mathrm{O}$ stars play a very important role. Their effective temperatures are so high that they interact with the surrounding interstellar matter and trigger or stop the formation of other stars.

* Operated by Associated Universities, Inc., under contract with the National Science Foundation. 
In general, star formation may take place as follows. The interstellar gas consists of cool, dense clouds with temperatures of $60 \mathrm{~K}$ or less which are embedded in a hot, tenuous intercloud gas with temperatures of several thousand $\mathrm{K}$. These clouds are in pressure equilibrium until some process (e.g., a sudden compression by a density wave or a rapid cooling by an increased dust production) initiates their gravitational contraction. At this time a large fraction of the mass of the cloud is in the form of molecules and dust particles. The subsequent evolution of the cloud is not clear, but in any case it cannot be simple gravitational contraction and fragmentation (as is usually assumed), since all observations indicate that the most massive stars are formed last and that their formation is the most difficult task for nature. For example, there exist T-Tauri associations which do not contain early-type stars, and it is not at all clear that ultimately $\mathrm{O}$ stars will form there.* In $\mathrm{O}$ associations, star formation appears to take place in subgroups which contain about $2 \times 10^{3} M_{\odot}$. Once the $O$ stars of a subgroup are formed they ionize the remnant of the proto-subgroup forming what we now call a 'compact Hil region'. The mass ratio of ionized gas to stars in these subgroups is generally less than 10 percent, indicating a very high efficiency of the process of star formation.

As a rule of thumb, stars need about 1 per cent of their main-sequence lifetime for their pre-main-sequence contraction. This means that stars of lower mass, which have been formed together with $\mathrm{O}$ stars, should still be on their pre-main-sequence contraction while the $O$ stars have long since reached the main sequence. Compact young $\mathrm{H}$ II regions therefore are the obvious places to search for protostars. This, however, does not imply that protostars can only be found in Hil regions; the point is that we lack criteria for selecting regions where only stars of lower mass are formed.

In the following sections I will review observations of neutral condensations and protostars associated with $\mathrm{H}$ II regions. I will also include observations which may yield information on the original density distribution of the contracting protostar cluster.

\section{The Distribution of Ionized Gas Inside HII Regions}

The surface brightness of both optical and radio emission from an $\mathrm{HII}$ region is proportional to the emission measure which is the square of the electron density integrated along the line of sight. Therefore, these observations should be heavily weighted towards regions of high space density of the plasma. This is certainly correct for radio observations, but in optical observations the extinction by dust plays an intervening role. As a consequence only radio observations can give a correct picture of the surface-brightness distribution (and hence of the emission-measure distribution) of an $\mathrm{HII}$ region. However, only very recently did aperture-synthesis observations at radio wavelengths yield an angular resolution comparable to that of optical observations.

\footnotetext{
* See also Section 5 in the following discussion (Chapter 26) on a new mechanism for star formation. (Ed.)
} 
Single-dish radio observations with an angular resolution of a few minutes of arc reveal that most $\mathrm{H}$ II regions with high surface brightness consist of compact components with linear dimensions of less than $1 \mathrm{pc}$, which are embedded in extended lowdensity $\mathrm{H}$ II regions. The average density of the compact components is high, somewhere between $10^{2}$ and $10^{4} \mathrm{~cm}^{-3}$, but they contain only a few or a few times $10 M_{\odot}$ of ionized hydrogen. Probably these compact components represent the very early evolutionary stages of subgroups in $\mathrm{O}$ associations, whose exciting stars in most cases cannot be seen, probably since they are still hidden in a dense circumstellar dust cloud. Figure 1 shows radio contours of the Orion Nebula, observed with an angular resolution of $2^{\prime}$ (or $0.3 \mathrm{pc}$ at $450 \mathrm{pc}$ distance), superimposed on a photograph (Schraml and Mezger, 1969). The Orion Nebula consists of the two compact components M42 and M43. We think we know the exciting stars for these two compact H II regions. The obscuration by dust is relatively low; note, however, the dark bay across M42 which extends close to the Trapezium. I will refer to this dust cloud later in my Report.

Several lines of investigation have shown that in the Orion Nebula (and also in other HII regions) the ionized gas is clumped on an even smaller scale, of angular

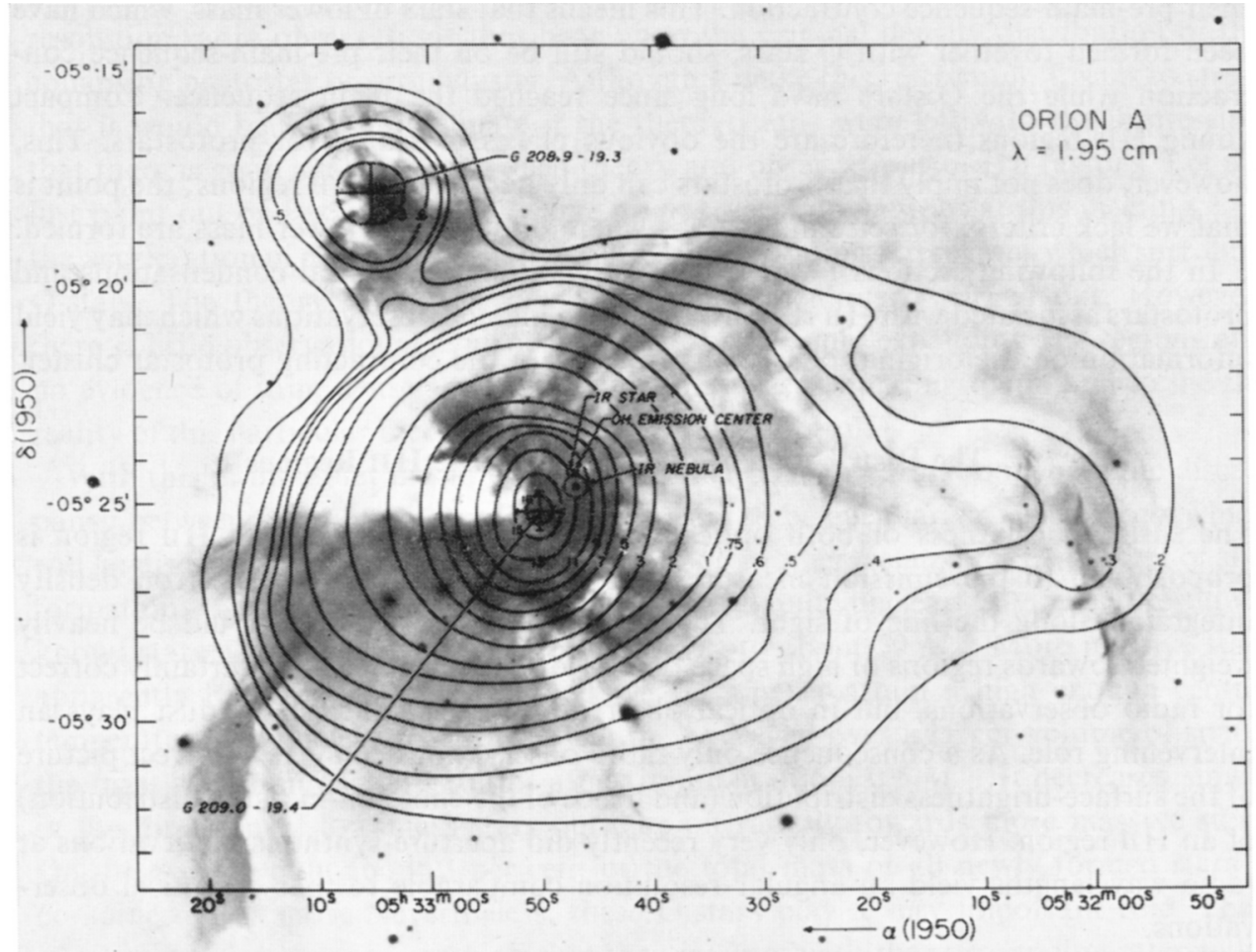

Fig. 1. Overlay of the radio continuum contours, observed with an angular resolution of $2^{\prime}$ at $\lambda=2 \mathrm{~cm}$, on a photograph of the Orion Nebula (Schraml and Mezger, 1969). The source in the upper half of the Figure (G 208.9-19.3) is M43; the source in the lower half (G 209.0-19.4) is M42. 

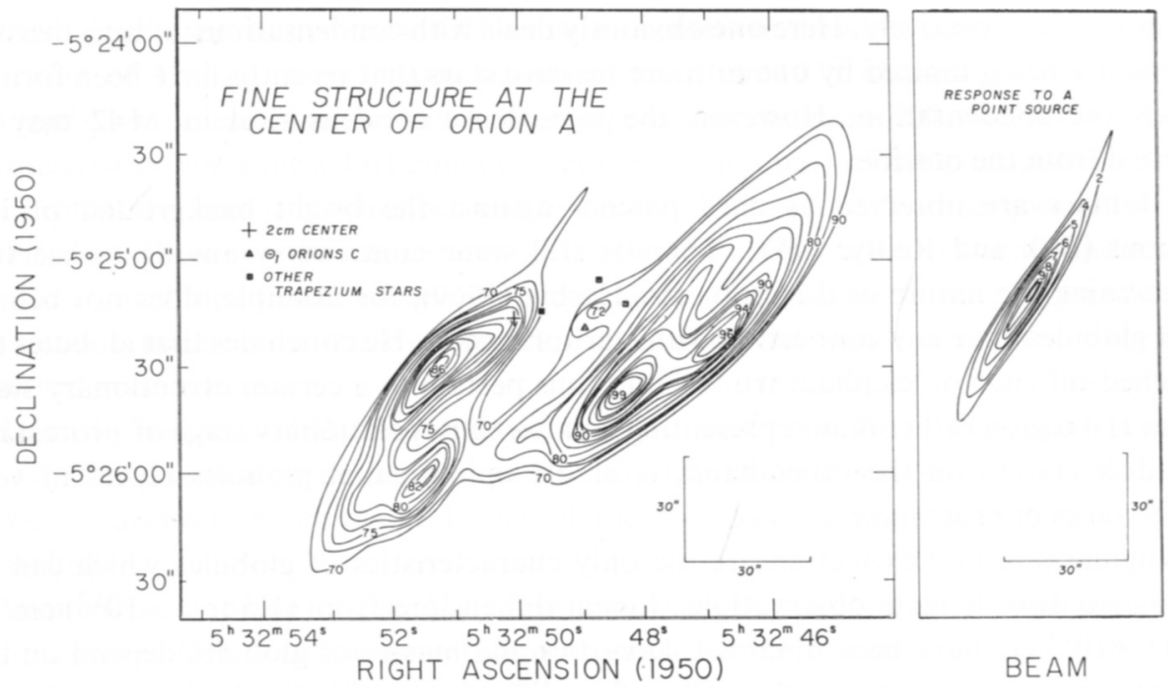

Fig. 2. Aperture synthesis map of the continuum radiation of the compact component $M 42$ in the Orion Nebula, observed at $\lambda=11 \mathrm{~cm}$ with an angular resolution of about $8^{\prime \prime}$ in right ascension (Webster and Altenhoff, 1970).

dimensions $3^{\prime \prime}$ to $10^{\prime \prime}$, linear dimensions $1.5 \times 10^{-2} \mathrm{pc}$ to $5 \times 10^{-2} \mathrm{pc}$. Webster and Altenhoff (1970) have observed some such condensations directly, using the National Radio Astronomy Observatory three-element interferometer. Figure 2 shows their result for M42. At least four, perhaps five, superdense condensations are found with angular dimensions of about 4". Adopting a distance of the Orion Nebula of $450 \mathrm{pc}$, we obtain $\left\langle n_{e}^{2}\right\rangle^{1 / 2}=5 \times 10^{4} \mathrm{~cm}^{-3}, M_{\mathrm{HII}} / M_{\odot}=2 \times 10^{-3}$ and linear dimensions of about $1.6 \times 10^{-2} \mathrm{pc}\left(=5 \times 10^{16} \mathrm{~cm}=3200 \mathrm{AU}\right)$. This is about the diameter of a protostar in its earliest evolutionary stages, where its average density is somewhere between $10^{6}$ and $10^{8} \mathrm{~cm}^{-3}$. It has been shown by Hjellming (1969) that such a protostar, exposed to the Lyman continuum flux from an $\mathrm{O}$ star, will only be ionized superficially and that it can easily survive during the lifetime of an HII region $\left(\approx 10^{6} \mathrm{yr}\right)$. In fact the increased outer pressure may initiate the gravitational contraction of such a star. These superdense condensations contain only about 3 per cent of the total radio-flux density of M42. Further interferometer observations are planned to search for more condensations, which must have smaller angular dimensions and thus could be fragments with masses lower than that of stars.

In the case of M42 the superdense HII condensations do not coincide with very conspicuous optical features. However, in M8 the center of radio emission coincides with a very interesting optical feature, viz., two very bright nebulae of roughly $12^{\prime \prime}$ angular size, together known as the 'Hourglass Nebula'. The exciting O7 star, Herschel 36 , is embedded in a dust cloud. The two nebulae apparently contain several stellar components. Recently Smith and Weedman (1969) investigated the kinematics of the two nebulae and found that each of them expanded with velocities of 10 and 
$7 \mathrm{~km} \mathrm{sec}^{-1}$, respectively. Here one obviously deals with condensations of high density, which are being ionized by one or more massive stars that recently have been formed inside the condensation. However, the superdense condensation in M42 may be ionized from the outside.

Globules are observed as dark patches against the bright background of $\mathrm{HII}_{\mathrm{II}}$ regions (Bok and Reilly, 1947). There is still some controversy amongst observers concerning the nature of these objects. Herbig (1969), for example, does not believe that globules have any connection with star formation. He concludes that globules are pinched-off ends of 'elephant trunks' and thus pertain to a certain evolutionary stage of an $\mathrm{HII}$ region rather than representing a particular evolutionary stage of protostars. Reddish (1969), on the other hand, is of the opinion that globules represent very early stages of protostars.

Angular size and extinction are the only characteristics of globules which can be estimated directly from observations. Linear dimensions from $\left(1.5\right.$ to $\left.5 \times 10^{17}\right) \mathrm{cm}$ up to $15 \times 10^{17} \mathrm{~cm}$ have been obtained. Densities and masses of globules depend on the adopted gas-to-dust ratio, and it is therefore difficult to estimate whether or not these objects are in fact gravitationally unstable.

\section{Centers of Anomalous Radio Line Emission*}

It has been known for some time that sources of anomalous (i.e., nonthermal) $\mathrm{OH}$ emission often have very small linear dimensions ( $\ll 1 \mathrm{pc})$. This seems to hold also for emission sources of other, recently detected, radio lines $\left(\mathrm{H}_{2} \mathrm{O}, \mathrm{NH}_{3}, \mathrm{C}\right)$. We will call such small-scale sources 'emission centers', although we do not always imply that their dimensions are as small as in the $\mathrm{OH}$ case. The $\mathrm{OH}$ emission centers are classified as Class I, if the two center lines (at 1665 and $1667 \mathrm{MHz}$ ) are predominantly emitted. Class IIa and IIb pertain to the predominant emission of the $1720 \mathrm{MHz}$ and 1612 $\mathrm{MHz}$ line, respectively. At present, Class I emission centers are the best investigated; they show a tendency to be located towards the edge of compact Hil regions. Using the powerful VLB interferometer technique the MIT group (Moran et al., 1968) succeeded in resolving some of these Class I emission centers. As an example, we consider the $\mathrm{OH}$ emission spectrum in IC 1795 (W3). Observed with a single dish, it consists of a number of spikes which are either right- or left-hand circularly polarized. Taking advantage of the frequency shift and different polarization characteristics of the individual spikes the MIT group showed that each individual spike in the spectrum corresponds to an individual emission center. The projected positions of seven emission centers lie in an area of roughly $1.2^{\prime \prime} \times 2.3^{\prime \prime}$. Only one center has a simple disk shape of apparent diameter $0.005^{\prime \prime}$. The others show structure and might be clusters of two or three emission centers. In one case the observations indicate an elongated structure. At a distance of $2.5 \mathrm{kpc}$ adopted for IC 1795, an angle of $0.005^{\prime \prime}$ corresponds to a linear size of $2 \times 10^{14} \mathrm{~cm}=13 \mathrm{AU}$. Mezger and Robinson (1968) investigated the

* See also the Report by Weaver, p. 22. (Ed.) 
physical characteristics of these $\mathrm{OH}$ emission centers and estimated that densities of the order of $10^{11}$ atom $\mathrm{cm}^{-3}$ and masses of less than $10^{-2} M_{\odot}$ are involved in the $\mathrm{OH}$ emission; these objects may have temperatures between 200 and $500 \mathrm{~K}$. Such characteristics place these $\mathrm{OH}$ emission centers on that part of the evolutionary track of a protostar where it just has become opaque and subsequently undergoes adiabatic contraction. However, the masses involved in the $\mathrm{OH}$ emission are too small, and it was therefore concluded that the emission occurs in the outer layers of the protostar only. It is still a puzzle how the over-population is achieved which is necessary for the observed maser effect.

Recently Wilson and Barrett (1968) discovered that Class IIb OH emission centers are associated with a particular type of IR star and exhibit a very strong maser effect. However, accurate positions and sizes of these emission centers have not yet been published.

Anomalous emission of the $22.2 \mathrm{GHz}$ line of $\mathrm{H}_{2} \mathrm{O}$ usually comes from the same regions where $\mathrm{OH}$ emission centers are observed. In two objects of Class IIb, viz., VY CMa and IRC +10406 , the $\mathrm{H}_{2} \mathrm{O}$ emission features are flanked on either side by a range of velocities of $\mathrm{OH}$ emission, and there is no $\mathrm{OH}$ emission in the velocity range of $\mathrm{H}_{2} \mathrm{O}$ emission (Knowles et al., 1969; Turner et al., 1970). VLB observations of the $\mathrm{H}_{2} \mathrm{O}$ emission centers have been unsuccessful to date, but the time variation of the $\mathrm{H}_{2} \mathrm{O}$ emission occurs with a much shorter time scale than the time variation of $\mathrm{OH}$ emission; from this an upper limit of $10^{16} \mathrm{~cm}$ can be inferred on the size of the $\mathrm{H}_{2} \mathrm{O}$ emission centers (Knowles et al., 1969).

$\mathrm{NH}_{3}$ emission, to date observed only in two sources close to the galactic center, shows an irregular distribution over an area about $10^{\prime}$ wide, with considerable variation in density, velocity, and state of excitation. Kinetic temperatures of the gas are mainly in the range (20 to $100 \mathrm{~K}$ ) (Cheung et al., 1969).

A major step forward in the investigation of $\mathrm{HII}$ regions was made through observations of radio recombination lines. In the course of observations of the Hel09 $\alpha$ lines, Palmer et al. (1967) discovered a new and unexpected recombination line which was subsequently identified as that of carbon. Figure 3 shows the $109 \alpha$ recombination spectrum in the Orion Nebula; one recognizes the recombination lines of both $\mathrm{He}$ and C. The $\mathrm{C} 109 \alpha$ line is much narrower than both $\mathrm{H}$ and $\mathrm{He}$ lines, thus indicating that it must be emitted from regions which are considerably cooler than the hydrogen plasma of the $\mathrm{H}$ II region. The intensity of that line, on the other hand, is much stronger than would be expected from the normal abundance ratio, and there are further observational indications that the carbon line is also amplified by some sort of maser effect. An investigation of the emission characteristics of the carbon line is very cumbersome and time consuming. It was first done for NGC 2024 by Zuckerman and Palmer (1968) and subsequently for the Orion Nebula (M42) by Churchwell and Mezger (1969). The results were in both cases similar: the line emission appears to come from narrowly confined regions which do not coincide with the center of the associated $\mathrm{H}_{\mathrm{II}}$ regions. In the case of M42, at least one carbon-line emission center appears to coincide with the upper end of the dark bay, a dust lane which obscures part of the 


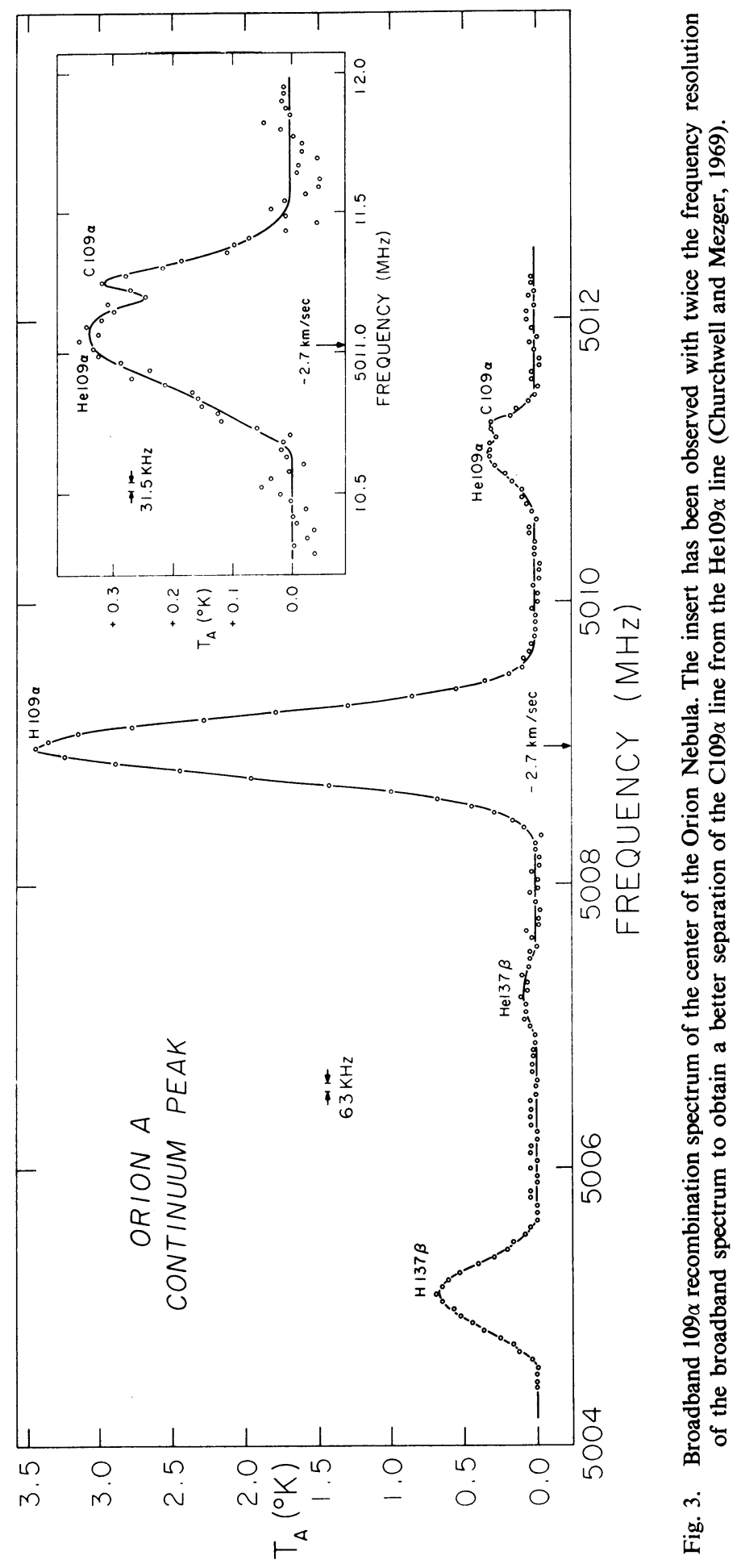


optical emission. This result supports the hypothesis put forward by Zuckerman and Palmer (1968) that the carbon-line emission comes from dense dust clouds opaque to the Lyman-continuum UV flux but transparent to the UV radiation between 912 and $1100 \AA$ which is capable of ionizing carbon. [See also in the following discussion, Chapter 26, Section 2 on the C line (Ed.)]

\section{IR Objects}

I mention IR objects here only for the sake of completeness and since it may help in the following discussion. There are obviously two different types of IR objects found in HII regions. First are the IR stars of the type observed first in the Orion Nebula by Becklin and Neugebauer (1967). The BN star in Orion has a color temperature of about $700 \mathrm{~K}$ and linear dimensions of about $4.4 \times 10^{14} \mathrm{~cm}(=30 \mathrm{AU})$. This object may be the nucleus of a protostar. Stars of this type are best observed in the wavelength range between 3 and $6 \mu$. Second are IR nebulae of much larger angular dimensions which are best observed at 10 and $20 \mu$. The first example of this kind was detected by Kleinmann and Low (1967) in M42, northwest of the Trapezium. At the moment the most probable interpretation of these objects is that they are dust clouds heated by starlight. The existence of circumstellar dust clouds has been inferred from a variety of independent observations (Reddish, 1967; Schraml and Mezger, 1969). Their existence implies that dust can survive for a relatively long time in Hil regions, that the dust grains have a high albedo in the UV, and that the dust does not expand as rapidly as the ionized gas. It follows that dust plays an even more important role in the formation of stars than is generally believed. Herbig's (1969) suggestion that dust is a by-product of star formation is of high interest in this context.

\section{A Summary of Different Observations Pertaining to the Compact HII Region G209.0-19.4 (M42) in the Orion Nebula}

In a paper given at the recent Liège Symposium (Mezger, 1969), I have tried to summarize and to correlate the observations of various neutral condensations and pre-main sequence stellar objects together with the distribution of ionized hydrogen in the compact HII region G209.0-19.4*, which coincides approximately with the Hil region M42 in the Orion Nebula. It may be of interest to mention the results here briefly.

Figure 4 shows a schematic representation of the positions and - where possible of the extension of the various observed objects. The hatched lines indicate the edges of the whole compact $\mathrm{HII}$ region which is embedded in a more extended low-density Hil region. The diameter of the whole region is $0.65 \mathrm{pc},\left\langle n_{e}^{2}\right\rangle^{1 / 2}=2.3 \times 10^{3} \mathrm{~cm}^{-3}$, and the total mass of ionized hydrogen $M_{\mathrm{H}_{\mathrm{II}}}=7 M_{\odot}$. Since the star density in this area is highest, the ratio $M_{\mathrm{H}_{\mathrm{II}}} / M_{*}$ must be considerably lower than the value 0.1 derived by Menon (1961) for the whole Orion Nebula. This reiterates that the efficiency of star

* This symbol summarizes the position of the radio source in galactic $(G)$ coordinates. (Ed.) 


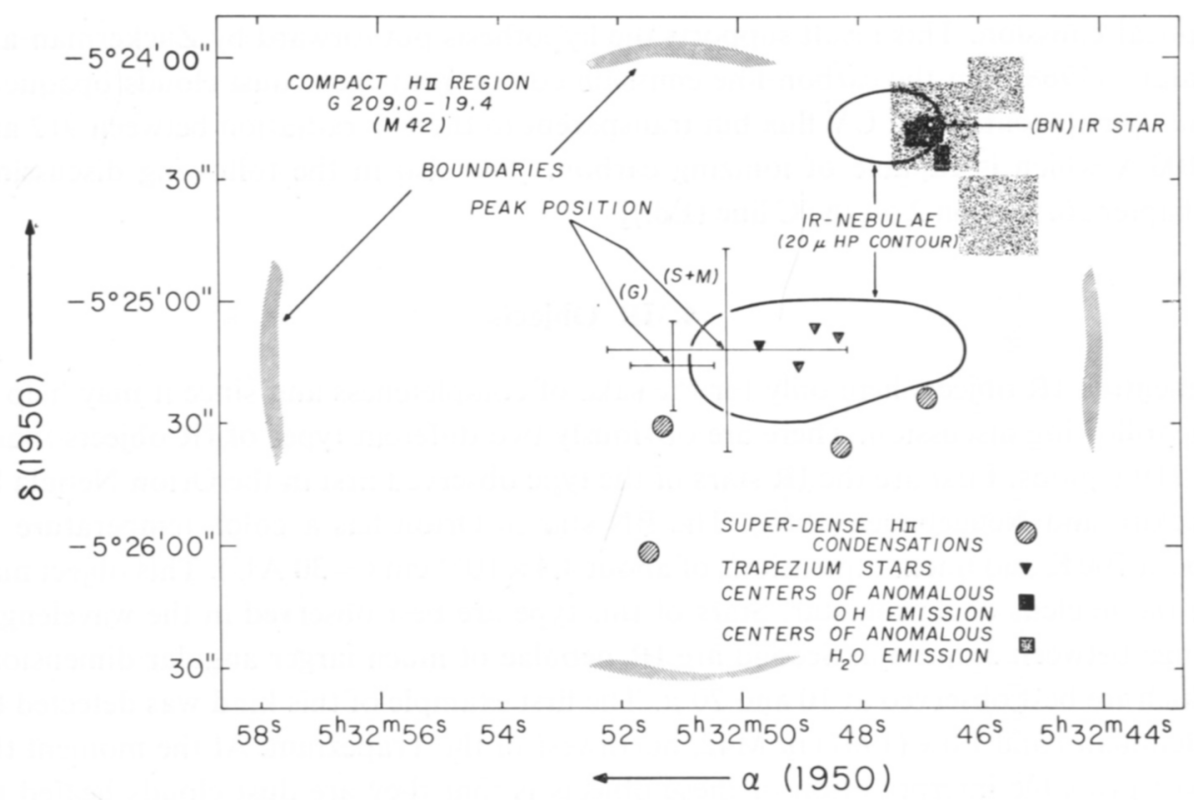

Fig. 4. Schematic representation of various objects observed in the compact Hil region M42 in the Orion Nebula (Mezger, 1969).

formation in subgroups is very high. The center of the radio free-free continuum does not coincide with the Trapezium but lies slightly to the east of it.

The positions and sizes of four superdense condensations of ionized hydrogen south of the Trapezium are shown (Webster and Altenhoff, 1970). A fifth condensation may be located close to the Trapezium. According to recent computations by Larson (1969) the outer layers of an evolving protostar consist of cool and neutral matter which undergoes a free-fall contraction until the protostar appears at the lower end of the Hayashi track. Such a protostar, if exposed to the Lyman continuum flux from a nearby $\mathrm{O}$ star, would be ionized in its outer layers but otherwise should not be affected in its evolution. It may be that these four superdense condensations are protostars in such a state; however we must await further observations with higher angular resolution. It has been pointed out by Osterbrock and Flather (1959) quite some time ago and recently confirmed independently by Hjellming and Churchwell (1969) that the clumping in the Orion Nebula must be very high. The ionized gas appears to occupy on the average only $\frac{1}{30}$ th of the total volume. We hope that further interferometer observations will tell us something about the size of these clumps.

Centers of anomalous emission of the $\mathrm{OH}$ and $\mathrm{H}_{2} \mathrm{O}$ lines have been found in an area northwest of the Trapezium. Their size is too small to be shown in the diagram. There are at least three different emission centers observed for each line. Positions of the $\mathrm{OH}$ emission centers have been obtained with an interferometer (Raimond and Eliasson, 1969); the $\mathrm{H}_{2} \mathrm{O}$ positions are obtained with a single-dish radio telescope and therefore less accurate (Meeks et al., 1969). The shaded areas indicate the positional 
uncertainties. In one case $\mathrm{OH}$ and $\mathrm{H}_{2} \mathrm{O}$ emission centers coincide with the Becklin and Neugebauer IR star within the positional uncertainties of the various objects.

Churchwell and Mezger (1969) have investigated the sources of emission of the carbon recombination line in M42. Although the reduction of these observations is not yet fully completed, it appears that emission comes from one, possibly from two different areas of not very large angular dimensions. One appears to be located SSE and the other one possibly NNE of the Trapezium. The latter could coincide with the upper end of a dust lane. The radial velocities of the carbon-line emitting regions deviate strongly from the mean radial velocity of the ionized hydrogen gas but are rather similar to that of both the surrounding neutral gas and the Trapezium stars.

The two extended nebulae observed in the far IR are shown by solid lines. According to Ney and Allen (1969), the amount of dust needed to account for the observed emission is rather small, $\lesssim 0.06 M_{\odot}$. These dust clouds may be the remnants of protostars or protoclusters.

The association of the BN star and the centers of $\mathrm{OH}$ and $\mathrm{H}_{2} \mathrm{O}$ emission northwest of the Trapezium may indicate a group of very young stellar objects. The fact that these objects are not associated with an increased continuum emission, similar to that of the superdense condensations, is somewhat puzzling. It may well be that these objects are located at quite a distance from the Trapezium stars and their apparent close association with the Trapezium is merely a projection effect.

\section{Acknowledgements}

I am indebted to W. Altenhoff, E. Churchwell, and W. Webster for permission to quote their unpublished results.

\section{References}

Becklin, E. E. and Neugebauer, G.: 1967, Astrophys. J. 147, 799.

Bok, B. J. and Reilly, E. F.: 1947, Astrophys. J. 105, 255.

Cheung, A. C., Rank, D. M., Townes, C. H., Knowles, S. H., and Sullivan III, W. T.: 1969, Astrophys. J. Lett. 157, L13.

Churchwell, E. and Mezger, P. G.: 1969, paper presented at the 130th American Astronomical Society Meeting, Albany, New York.

Herbig, G. H.: 1969, paper presented at the VIth international astrophysical symposium, Liège. Hjellming, R. M.: 1969, paper presented at the VIth international astrophysical symposium, Liège. Hjellming, R. M. and Churchwell, E.: 1969, Astrophys. Letters 4, 165.

Kleinmann, D. E. and Low, F. J.: 1967, Astrophys. J. Lett. 149, L1.

Knowles, S. H., Mayer, C. H., Cheung, A. C., Rank, D. M., and Townes, C. H.: 1969, Science 163, 1055.

Larson, R. B.: 1969, Monthly Notices Roy. Astron. Soc. 145, 405.

Meeks, M. L., Carter, J. C., Barrett, A. H., Schwartz, P. R., Waters, J. W., and Brown III, W. E.: 1969, Science 165, 180.

Menon, T. K.: 1961, Publ. Nat. Radio Astron. Observ. 1, 1.

Mezger, P. G.: 1969, paper presented at the VIth international astrophysical symposium, Liège. Mezger, P. G. and Robinson, B. J.: 1968, Nature 220, 1107.

Moran, J. M., Burke, B. F., Barrett, A. H., Rogers, A. E. E., Ball, J. A., Carter, J. C., and Cudaback, D. D.: 1968, Astrophys. J. Lett. 152, L97.

Ney, E. D. and Allen, D. A.: 1969, Astrophys. J. Lett. 155, L193. 
Osterbrock, D. E. and Flather, E.: 1959, Astrophys. J. 129, 26.

Palmer, P., Zuckerman, B., Penfield, H., Lilley, A. E., and Mezger, P. G.: 1967, Nature 215, 40.

Raimond, E. and Eliasson, B.: 1969, Astrophys. J. 155, 817.

Reddish, V. C.: 1967, Monthly Notices Roy. Astron. Soc. 135, 251.

Reddish, V. C.: 1969 , paper presented at the VIth international astrophysical symposium, Liège. Schraml, J. and Mezger, P. G.: 1969, Astrophys. J. 156, 269.

Smith, M. G. and Weedman, D. W.: 1969, paper given at the 130th American Astronomical Society Meeting, Albany, New York.

Turner, B. E., Buhl, D., Churchwell, E., Mezger, P. G., and Snyder, L. E.: 1970, Astron. Astrophys. 4, 165.

Webster, W. J. and Altenhoff, W.: 1970, Astrophys. Lett. 5, 233.

Wilson, W. J. and Barrett, A. H.: 1968, Science 161, 778.

Zuckerman, B. and Palmer, P.: 1968, Astrophys. J. Lett. 153, L145. 